\title{
On the non-existence of certain group topologies
}

\author{
by \\ Christian Rosendal (Pasadena, CA, and Urbana, IL)
}

\begin{abstract}
Minimal Hausdorff (Baire) group topologies of certain groups of transformations naturally occurring in analysis are studied. The results obtained are subsequently applied to show that, e.g., the homeomorphism groups of the rational and of the irrational numbers carry no Polish group topology. In answer to a question of A. S. Kechris it is shown that the group of Borel automorphisms of $\mathbb{R}$ cannot be a Polish group either.
\end{abstract}

1. Introduction. The question of which group topologies different groups can have is of course of interest to many branches of mathematics, but for descriptive set theory this question usually takes a specific form, namely, can a particular group be equipped with a Polish, i.e., a separable completely metrisable, group topology? This question is also tightly connected with the possible "uniqueness" of a given Polish group topology. Of course the fundamental results by R. M. Solovay [19] and their refinements by S. Shelah [16] show that it is consistent with the axioms of set theory minus the axiom of choice that any Polish group has a unique Polish group topology. On the other hand, using a Hamel basis (and thus some amount of $\mathrm{AC}$ ) one easily constructs a multitude of Polish group topologies on $(\mathbb{R},+)$. Actually, the situation is even worse, for the abstract groups $(\mathbb{R},+)$ and $\left(\mathbb{R}^{2},+\right)$ are isomorphic and thus $(\mathbb{R},+)$ can be retopologised as $\left(\mathbb{R}^{2},+\right)$, and thus as a Polish group non-isomorphic to itself. The question then boils down to: When does the group structure exclude these constructions involving the axiom of choice?

One basic result in this area is the fundamental result on automatic continuity of homomorphisms by R. M. Dudley [3]. It apparently passed rather unnoticed, despite the fact that since its publication a number of questions and answers have appeared covering only specific subcases of his theorem. We can do no better than refer to the original article, but let us

2000 Mathematics Subject Classification: Primary 03E15.

Key words and phrases: group topology, automorphism group, homeomorphism group, category algebra, Borel equivalence relation. 
just mention the special case of his theorem stating that any homomorphism from a complete metric group into a free group is necessarily continuous with respect to the discrete topology on the free group. Thus, by consequence, the free group on a continuum of generators carries no Polish group topology and, e.g., any homomorphism from a Polish group into $\left(\mathbb{Z}^{n},+\right)$ is continuous.

A problem of Ulam, number 96 in the Scottish Book [13], asks whether $\mathcal{S}_{\infty}$, the group of all permutations of the natural numbers, can be made into a locally compact Polish group. This was solved in the negative by E. Gaughan [5], who actually showed that any Hausdorff group topology on $\mathcal{S}_{\infty}$ extends the usual Polish group topology of pointwise convergence on the discrete space $\mathbb{N}$. Recently, A. S. Kechris and the author proved in [12] that any homomorphism from $\mathcal{S}_{\infty}$ into a separable group is continuous, which, in combination with Gaughan's result, implies that $\mathcal{S}_{\infty}$ has only one non-trivial separable group topology. Actually, we shall see that the answer to Ulam's problem is even more striking, namely, there is no non-trivial homomorphism from $\mathcal{S}_{\infty}$ into a locally compact Polish group, and, moreover, this also holds for many other groups of countable structures.

We classify minimal Hausdorff group topologies of some automorphism groups of boolean algebras, using quite standard methods, and apply these results to prove the non-existence of Polish group topologies of several groups occurring in descriptive set theory and analysis, e.g., the homeomorphism groups of the irrational and of the rational numbers. We should mention that in a series of papers R. Kallman (see [10]) has proved that a number of, in particular, homeomorphism groups have a unique, if any, Polish group topology.

We also prove that the group of Borel automorphisms of $\mathbb{R}$ cannot have a second countable Hausdorff group topology. For a detailed study of a number of different topologies on this group one can consult the recent article by S. Bezuglyı̆, A. H. Dooley and J. Kwiatkowski [1].

I would like to thank Alekos Kechris for initially getting me interested in the subject and Ben Miller for many discussions on this and other matters. But mostly I am indebted to the anonymous referee for making this a hopefully more readable paper.

2. Polish group topologies. Let us begin with something simple. By the Borel isomorphism theorem all uncountable standard Borel spaces are isomorphic to $\mathbb{R}$, so all groups of Borel automorphisms of uncountable standard Borel spaces are naturally isomorphic. The following answers a question posed to the author by A. Kechris:

THEOREM 1. There is no second countable Hausdorff topology on the group of Borel automorphisms of $\mathbb{R}$. 
Proof. Assume $G=\operatorname{Aut}\left(\mathbb{R}, \boldsymbol{\Delta}_{1}^{1}\right)$ is given a Hausdorff group topology. Suppose $A \subseteq \mathbb{R},|A|>2$ and let $G_{(A)}=\{g \in G \mid \operatorname{supp}(g) \cap A=\emptyset\}$. We claim that $G_{(A)}$ is closed in $G$. For $x, y \in \mathbb{R}$, denote by $(x y)$ the transposition of $x$ with $y$.

Obviously, if $g \in G_{(A)}$ and $x, y \in A$, then $(x y) \cdot g=g \cdot(x y)$.

Conversely, suppose that $g \cdot x \neq x$ for some $x \in A$. Then there is $y \in A$ such that $y \neq g \cdot x, x$, whereby $g \cdot(x y) \cdot x=g \cdot y \neq g \cdot x=(x y) \cdot g \cdot x$ and thus $(x y) \cdot g \neq g \cdot(x y)$. So

$$
G_{(A)}=\bigcap_{x, y \in A}\{g \in G \mid(x y) \cdot g=g \cdot(x y)\},
$$

and, as the topology on $G$ is Hausdorff, this is an intersection of closed sets, so closed.

Now fix $x \in \mathbb{R}$ and suppose that $A \neq B \subseteq \mathbb{R}$ are sets of cardinality $>2$ with $x \notin A, x \notin B$. Find, e.g., $y \in A \backslash B$ and notice that $(x y) \in G_{(B)} \backslash G_{(A)}$, i.e., $G_{(A)} \neq G_{(B)}$. So we see that there are $2^{2^{\aleph_{0}}}$ distinct closed subgroups of $G$ and hence the topology cannot be second countable.

The same proof shows that if we identify two Borel automorphisms if they agree on a co-countable set, then we still have the same conclusion. For instead of using transpositions, we can use Borel automorphisms switching uncountable Borel sets, noting that $\mathbb{R}$ is Borel isomorphic to $\mathbb{R} \times \mathbb{R}$.

For $\mathfrak{B}$ a boolean algebra and $g \in \operatorname{Aut}(\mathfrak{B})$, we let $\operatorname{stab}(g)=\{a \in \mathfrak{B}$ $\forall b \leq a(g(b)=b)\}$.

Suppose $\mathcal{M}$ is any structure and $H=\operatorname{Aut}(\mathcal{M})$ is its group of automorphisms. Then $H$ is naturally equipped with a group topology having as subbasis the sets $\{h \in H \mid h(a)=b\}$, where $a$ and $b$ run over the elements of $\mathcal{M}$. We call this the topology of pointwise convergence on $\mathcal{M}$ (think of $\mathcal{M}$ as a discrete topological space).

We are now ready for our main result from which more interesting corollaries will follow:

Proposition 2. Suppose $\mathfrak{B}$ is a boolean algebra and $G \leq \operatorname{Aut}(\mathfrak{B})$ satisfies

(i) $\forall a>0 \exists a_{0}, a_{1}>0 \exists k \in G\left(a_{0} \wedge a_{1}=0\right.$ \& $a_{0}, a_{1} \leq a \& \neg a \in$ $\left.\operatorname{stab}(k) \& k\left(a_{i}\right)=a_{1-i}\right)$.

(ii) $\forall a \leq b<1 \exists k \in G(k(a)=a \& k(b \wedge \neg a) \wedge b=0)$.

(iii) $G$ acts transitively on $\mathfrak{B} \backslash\{0,1\}$.

Assume moreover that $\mathfrak{B}$ has a countable dense subalgebra $\mathfrak{A}$ and $G$ is a Hausdorff, Baire, topological group. Then the topology on $G$ extends the topology of pointwise convergence on $\mathfrak{B}$. 
We mention that if $\mathfrak{B}$ is a homogeneous (non-trivial) boolean algebra having a countable dense subalgebra and $G=\operatorname{Aut}(\mathfrak{B})$, then the conditions of Proposition 2 are satisfied.

Let us list the corollaries:

Corollary 3. There is no Polish group topology on the homeomorphism group of the rational numbers nor on the homeomorphism group of the irrational numbers.

COROLlary 4. The pointwise topology on the automorphism group of the countable atomless boolean algebra is the coarsest Hausdorff, Baire, group topology.

Corollary 4 is certainly imminent from Proposition 2 , as we can take $\mathfrak{B}$ to be the countable atomless boolean algebra and $G$ to be its automorphism group.

Proof of Corollary 3. We first prove it for the homeomorphism group $\mathrm{H}(\mathcal{N})$ of the irrationals. Let $\mathfrak{B}=\mathrm{CO}(\mathcal{N})$ be the algebra of clopen subsets of $\mathcal{N}$ and notice that there is a canonical representation of $\mathrm{H}(\mathcal{N})$ in $\operatorname{Aut}(\mathfrak{B}):$ an element of $\mathrm{H}(\mathcal{N})$ acts by translation on the clopen subsets of $\mathcal{N}$ and thus acts on $\mathfrak{B}$ by automorphisms. We claim that the representation is faithful, i.e., that different homeomorphisms have different representations. So suppose $f \neq g \in \mathrm{H}(\mathcal{N})$. Then for some $x \in \mathcal{N}, g(x) \neq f(x)$ and we can find a clopen set $C \subseteq \mathcal{N}$ with $x \in C$ such that $g " C \cap f^{\prime \prime} C=\emptyset$. Hence $f$ and $g$ have distinct representations in $\operatorname{Aut}(\mathfrak{B})$.

We now claim that $G=\mathrm{H}(\mathcal{N}) \leq \operatorname{Aut}(\mathfrak{B})$ satisfies (i)-(iii) in Proposition 2. This is essentially a consequence of the Aleksandrov-Urysohn theorem (see (7.7) in [11]). In particular, this theorem implies that any two non-empty clopen subsets of the irrationals are homeomorphic. So given any two clopen subsets $\emptyset \neq A, B \subsetneq \mathcal{N}$ there is a homeomorphism $k$ of $\mathcal{N}$ such that $k " A=B$, thus proving (iii). Now, notice that given a non-empty clopen set $A \subseteq \mathcal{N}$ there is a non-trivial partition of $A$ into clopen $A_{0}, A_{1}$ and there is a $k \in \mathrm{H}(\mathcal{N})$ such that $k$ is the identity on $\complement A$ and $k$ " $A_{0}=A_{1}$, $k " A_{1}=A_{0}$, hence proving (i). Similar reasoning verifies (ii).

Since $\mathcal{N}$ is second countable and zero-dimensional, we also see that $\mathfrak{B}$ has a countable dense subalgebra generated by a countable basis of clopen sets. So by the proposition, any Polish group topology on $\mathrm{H}(\mathcal{N})$ extends the topology of pointwise convergence on $\mathfrak{B}$, i.e., for any clopen $C \subseteq \mathcal{N}$, the subgroup $\{g \in \mathrm{H}(\mathcal{N}) \mid g " C=C\}$ is open. Now take a countable partition of $\mathcal{N}$ into non-empty clopen subsets $N_{n} \subseteq \mathcal{N}$ and find for each $\alpha \in 2^{\mathbb{N}}$ a $g_{\alpha} \in \mathrm{H}(\mathcal{N})$ such that

$$
g_{\alpha} " N_{0}=\bigcup_{\alpha(n)=1} N_{n+2} \cup N_{0}
$$


Then $g_{\alpha} " N_{0} \neq g_{\beta} " N_{0}$ for all $\alpha \neq \beta$ and the non-empty open subsets

$$
\left\{f \in \mathrm{H}(\mathcal{N}) \mid f " N_{0}=g_{\alpha} " N_{0}\right\}=g_{\alpha} \cdot\left\{f \in \mathrm{H}(\mathcal{N}) \mid f " N_{0}=N_{0}\right\}
$$

are all disjoint, contradicting the separability of $\mathrm{H}(\mathcal{N})$.

For $\mathrm{H}(\mathbb{Q})$ the argument is essentially identical. Again we let $\mathfrak{B}=\mathrm{CO}(\mathbb{Q})$ and $G=\mathrm{H}(\mathbb{Q}) \leq \operatorname{Aut}(\mathfrak{B})$. Since $\mathbb{Q}$ is zero-dimensional we see that the representation is faithful and (i)-(iii) follow as before, using the FréchetSierpiński theorem (see (7.12) in [11]) instead of the Aleksandrov-Urysohn theorem. Again this implies that all clopen subsets of $\mathbb{Q}$ are homeomorphic and as $\mathbb{Q}$ is second countable, $\mathfrak{B}$ has a countable dense subalgebra. Finally, notice that $\mathbb{Q}$ has a countable partition into non-empty clopen pieces using, e.g., irrational Dedekind cuts. So we can end the proof as before.

Definition 5. The uniform Suslin number of a topological group is the least infinite cardinal $\kappa$ such that there are not $\kappa$ disjoint left translates of any non-empty open set. This is equivalent to saying that any non-empty open set covers the group by $<\kappa$ left translates.

We then see that in Corollary 3, we actually have the stronger conclusion that any Hausdorff, Baire, group topology cannot have uniform Suslin number $\leq 2^{\aleph_{0}}$.

Proof of Proposition 2. The proof has three main steps: We first show that if $c \in \mathfrak{B}$, the set $\{g \in G \mid c \in \operatorname{stab}(g)\}$ is closed. Secondly, we prove that if $a, b \in \mathfrak{B}$, then the set $\{g \in G \mid b \leq g(a)\}$ is closed. And thirdly, using the existence of a countable dense subalgebra, we find some $c \in \mathfrak{B} \backslash\{0,1\}$ such that $G_{(c)}=\{g \in G \mid g(c)=c\}$ is an open subgroup.

We can assume that $\mathfrak{B} \neq\{0,1\}$. Suppose first that $c \in \mathfrak{B}, g \in G$ and $c \notin \operatorname{stab}(g)$. Then we can find $b \leq c$ such that $g(b) \neq b$, whereby either $b \wedge \neg g(b) \neq 0$ or $g(b) \wedge \neg b \neq 0$. Obviously, if $b \wedge \neg g(b) \neq 0$, there is $0<a \leq c$ such that $a \wedge g(a)=0$. And if $g(b) \wedge \neg b \neq 0$, then for $a=g^{-1}(g(b) \wedge \neg b) \leq b \leq c$ we also have $a \wedge g(a) \leq b \wedge(g(b) \wedge \neg b)=0$. So by (i), we can find $a_{0}, a_{1}>0, a_{0} \wedge a_{1}=0, a_{0}, a_{1} \leq a$ and $k \in G$ such that $\neg c \leq \neg a \in \operatorname{stab}(k)$ and $k\left(a_{i}\right)=a_{1-i}$. Since $a \wedge g(a)=0$, also $g\left(a_{0}\right) \leq g(a) \leq \neg a \in \operatorname{stab}(k)$ and thus $k \cdot g\left(a_{0}\right)=g\left(a_{0}\right) \neq g\left(a_{1}\right)=g \cdot k\left(a_{0}\right)$, whence $g$ and $k$ do not commute.

Conversely, if $c \in \operatorname{stab}(g)$ and $\neg c \in \operatorname{stab}(k)$, then clearly $g$ and $k$ commute. Thus

$$
c \in \operatorname{stab}(g) \Leftrightarrow g \in \bigcap_{\substack{\neg c \in \operatorname{stab}(k) \\ k \in G}}\{f \in G \mid f k=k f\} .
$$

Hence as $G$ is Hausdorff, for each $k \in G$ the set $\{f \in G \mid f k=k f\}$ is closed and thus for each $c \in \mathfrak{B}$ the set $\{g \in G \mid c \in \operatorname{stab}(g)\}$ is an intersection of closed sets and therefore closed itself. 
Notice now that for any $g, k \in G$, we have $\operatorname{stab}\left(g k g^{-1}\right)=g \cdot \operatorname{stab}(k)$. Suppose $b \leq g(a)$ and $a \in \operatorname{stab}(k)$. Then $g(a) \in g \cdot \operatorname{stab}(k)=\operatorname{stab}\left(g k g^{-1}\right)$ and $b \in \operatorname{stab}\left(g k g^{-1}\right)$. Conversely, if $b \not \leq g(a)$, then $g^{-1}(b) \not \leq a$ and there are by (i) some $0<b_{0}, b_{1} \leq g^{-1}(b) \wedge \neg a, b_{0} \wedge b_{1}=0$ and $k \in G$ such that $k\left(b_{i}\right)=b_{1-i}$ and $a \in \operatorname{stab}(k)$. That is, $g^{-1}(b) \notin \operatorname{stab}(k)$ and $a \in \operatorname{stab}(k)$, whereby $b \notin g \cdot \operatorname{stab}(k)=\operatorname{stab}\left(g k g^{-1}\right)$. Therefore,

$$
b \leq g(a) \Leftrightarrow g \in \bigcap_{a \in \operatorname{stab}(k)}\left\{f \in G \mid b \in \operatorname{stab}\left(f k f^{-1}\right)\right\} .
$$

Thus for each $a$ and $b$ the set $\{g \in G \mid b \leq g(a)\}$ is again an intersection of closed sets, so closed itself.

We suppose now that $0<a, b<1$ are some arbitrary fixed elements of $\mathfrak{B}$ and notice that

$$
b \not \leq g(a) \Leftrightarrow g^{-1}(b) \not \leq a \Leftrightarrow \exists c \in \mathfrak{A} \backslash\{0,1\}\left(c \leq g^{-1}(b) \& c \wedge a=0\right) .
$$

So the open set $\{g \in G \mid b \not \leq g(a)\}$ is the countable union of the closed sets $\left\{g \in G \mid c \leq g^{-1}(b)\right\}$, where $c \in \mathfrak{A} \backslash\{0,1\}$ and $c \wedge a=0$. Thus, as $G$ is Baire, one of these sets must have non-empty interior, say $V=\operatorname{int}\{g \in G \mid$ $\left.c \leq g^{-1}(b)\right\} \neq \emptyset$ for some fixed $c$.

Choose some $f \in V$, let $d=f^{-1}(b)$, and notice that $c \leq f^{-1}(b)=d<1$ and that $f^{-1} \cdot V$ is an open neighbourhood of the identity $1_{G}$ in $G$. Moreover, for any $h=f^{-1} g \in f^{-1} \cdot V, h(c)=f^{-1} g(c) \leq f^{-1}(b)=d$. Using (ii), we choose $k \in G$ such that $k(c)=c$ and $k(d \wedge \neg c) \wedge d=0$. Then for any $h \in f^{-1} \cdot V$,

$$
k h k^{-1}(c)=k h(c) \leq k(d) \leq c \vee \neg d .
$$

Therefore, if $h \in W:=\left(f^{-1} \cdot V\right) \cap\left(k f^{-1} \cdot V \cdot k^{-1}\right)$, we have $h(c) \leq d \wedge(c \vee \neg d)$ $=c$. But $W$ is easily seen to be an open neighbourhood of $1_{G}$ and if $h \in$ $W \cap W^{-1}$, then $h(c)=c$, which shows that the subgroup $G_{(c)}=\{g \in G \mid$ $g(c)=c\}$ contains the open set $W \cap W^{-1}$, so must be open in $G$.

Assume now that $0<e<1$ is any other element of $\mathfrak{B}$. Then as $G$ acts transitively on $\mathfrak{B} \backslash\{0,1\}$, there is an $h \in G$ with $h(c)=e$ and thus $G_{(e)}=h \cdot G_{(c)} \cdot h^{-1}$ is open in $G$.

This shows that for any element $e \in \mathfrak{B}$ the pointwise stabiliser, $G_{(e)}$, is open in $G$ and therefore, as any open subgroup is closed, also clopen, whence the topology on $G$ extends the pointwise topology induced by $\mathfrak{B}$.

For a set $A$ we let $A^{<\mathbb{N}}$ be the descriptive set-theoretic tree of all finite sequences of elements of $A$ under the relation of end-extension. $\operatorname{Aut}\left(A^{<\mathbb{N}}\right)$ is the group of permutations of the tree preserving the relation of endextension and therefore also the root (the empty sequence). Aut $\left(A^{<\mathbb{N}}\right)$ is naturally isomorphic to the group of isometries of $A^{\mathbb{N}}$, when the latter is equipped with its usual ultra-metric. 
By methods very similar to the proof of Proposition 2 one can show that any Hausdorff, Baire topology on $\operatorname{Aut}\left(\mathbb{N}^{<\mathbb{N}}\right)$ must extend the topology of pointwise convergence on $\mathbb{N}<\mathbb{N}$. Moreover, any Hausdorff topology on $\operatorname{Aut}\left(2^{<\mathbb{N}}\right)$ must likewise extend the topology of pointwise convergence on $2^{<\mathbb{N}}$.

In both cases one notices first that for any $s, t \in \mathbb{N}<\mathbb{N}$ the following set is closed:

$$
\left\{g \in \operatorname{Aut}\left(\mathbb{N}^{<\mathbb{N}}\right) \mid g(s)=t\right\} .
$$

Therefore as $g(s) \neq s \Leftrightarrow \exists t(\operatorname{length}(t)=\operatorname{length}(s) \& g(s)=t)$, by Baire one of the sets $\left\{g \in \operatorname{Aut}\left(\mathbb{N}^{<\mathbb{N}}\right) \mid g(s)=t\right\}$ must have non-empty interior and we continue as before. But in the case of $\operatorname{Aut}\left(2^{<\mathbb{N}}\right)$, we do not need the group to be Baire, as the existential quantifier above is over a finite set and any finite union of nowhere dense sets is nowhere dense in any topological space.

TheOREm 6. The group Aut $\left(\mathbb{N}^{<\mathbb{N}}\right)$ has a unique separable Hausdorff, Baire topology.

Proof. We know that any such topology must necessarily extend the pointwise topology. On the other hand, it was shown by Kechris and the author in [12] that any homomorphism from $\operatorname{Aut}(\mathbb{N}<\mathbb{N})$ with the pointwise topology into a separable group is automatically continuous, so this gives us the result.

TheOREm 7. Any Hausdorff topology on $\operatorname{Aut}\left(2^{<\mathbb{N}}\right)$ extends the topology of pointwise convergence.

3. Automorphism groups of the category algebra. We shall now see that certain groups of automorphisms of the category algebra of a perfect Polish space cannot be made into Polish groups. So let us first recall some basic facts about the category algebra.

Let $X$ be a Polish space. By $\operatorname{CAT}(X)$ we denote the boolean algebras of Borel sets modulo meagre sets. This algebra is called the category algebra of the space, and we notice that, as any Borel set is equivalent modulo a meagre set to a regular open set, the category algebra is canonically isomorphic to the complete boolean algebra of regular open sets $\mathrm{RO}(X)$ (recall that a set $U$ is called regular open in case it is equal to the interior of its closure). So in particular, any countable basis for the topology on the space, consisting of regular open sets, will generate a countable dense subalgebra of $\operatorname{CAT}(X)=\operatorname{RO}(X)$. It is easy to see that any two perfect Polish spaces have homeomorphic dense $G_{\delta}$ subsets, so the category algebras of perfect Polish spaces are all isomorphic. Thus, as non-empty regular open sets of 
perfect Polish spaces are perfect in themselves, we also see that the category algebra of a perfect Polish space is homogeneous.

A theorem due to Sikorski (see [11, 15.12]) implies that any isomorphism between the category algebras of two uncountable Polish spaces is induced by a Borel isomorphism between the underlying spaces. So the automorphism group of the category algebra of an uncountable Polish space can be identified with the group of Borel automorphisms preserving meagre sets, where two Borel automorphisms are identified if they agree on a comeagre set.

The theory of full groups was introduced by Henry Dye [4] in order to understand the orbit equivalence relation of a countable group of transformations on a Lebesgue space. This project has met with considerable success in different settings and it therefore seems natural to investigate the category analogue.

Let us first fix some notation. If $\mathrm{E}$ is an equivalence relation on a set $X, x$ is an element of $X$ and $A$ a subset of $X$, we let $[x]_{\mathrm{E}}$ be the equivalence class of $x, \complement A=X \backslash A$ and $[A]_{\mathrm{E}}$ the E-saturation of $A,[A]_{\mathrm{E}}=\{y \in X \mid \exists z \in A$ $(y \mathrm{E} z)\}$.

Definition 8. Suppose $X$ is a Polish space and $\mathrm{E}$ is a countable Borel equivalence relation on $X$, i.e., $\mathrm{E}$ is Borel and all its classes are countable.

- E is said to be generically ergodic if every E-invariant Borel set of $X$ is either meagre or comeagre.

- E is non-singular if the E-saturation of a meagre set is meagre.

Definition 9. Let $E$ be a non-singular countable Borel equivalence relation on a Polish space $X$. We let the Borel full group of $\mathrm{E}$ be defined by

$$
[\mathrm{E}]=\{g \mid g \text { is a Borel automorphism of } X \& \forall x \in X(g(x) \mathrm{E} x)\} .
$$

Let $N=\left\{g \in[\mathrm{E}] \mid \forall^{*} x \in X(g(x)=x)\right\}$, where $\forall^{*} x \in X$ means "for a comeagre set of $x \in X$ ". Then $N$ is a normal subgroup of [E] and we let the category full group of $\mathrm{E}$ be $[\mathrm{E}]^{*}=[\mathrm{E}] / N$.

As usual we will confuse cosets of $N$ with their representatives in [E]. Notice that by non-singularity, $[\mathrm{E}]^{*}$ is naturally a subgroup of $\operatorname{Aut}(\operatorname{CAT}(X))=$ $\operatorname{Aut}(\mathrm{RO}(X))$.

The following couple of basic lemmas will elucidate the structure of these groups.

LEMma 10. Let $\mathrm{E}$ be a non-singular countable Borel equivalence relation on a Polish space $X$ and assume that $A \subseteq X$ is comeagre. Then there is an E-invariant dense $G_{\delta}$ set $B \subseteq A$. 
Proof. Define by induction $A_{n}$ and $B_{n}$ by

- $A_{0}=A$.

- If $A_{n}$ is defined and comeagre, let $B_{n} \subseteq A_{n}$ be some dense $G_{\delta}$ subset.

- If $B_{n}$ is defined, let $A_{n+1}=\complement\left[\complement B_{n}\right]_{\mathrm{E}} \subseteq B_{n}$.

Then $A_{0} \supseteq B_{0} \supseteq A_{1} \supseteq B_{1} \supseteq \cdots$ and $B=\bigcap B_{n}=\bigcap A_{n}$ is both E-invariant and dense $G_{\delta}$.

Lemma 11. Let $\mathrm{E}$ be a non-singular countable Borel equivalence relation on a Polish space $X$ and let $\left\{g_{n}\right\}_{\mathbb{N}}$ be a countable set of elements of the Borel full group $[\mathrm{E}]$. Then there is an $\mathrm{E}$-invariant dense $G_{\delta}$ set $B \subseteq X$ such that each $g_{n}$ is a homeomorphism of $B$. Moreover, $B$ can be taken to be a subset of any prescribed comeagre set $Y \subseteq X$.

Proof. Let $\mathfrak{p}$ be the Polish topology of $X$. Since the $g_{n}$ are Borel automorphisms, there is a finer Polish topology $\tau$ on $X$, generating the same algebra of Borel sets, such that each $g_{n}$ is a homeomorphism of $(X, \tau)$. This follows quite trivially from Kuratowski's method of changing topologies (see, e.g., Chapter 13 in Kechris [11]), though I have not been able to locate an explicit proof other than in the case of a single automorphism (see Chapter 8 in Nadkarni [14], where the result is attributed to Mackey and Ramsay). Choose now a p-dense $G_{\delta}$ set $A \subseteq X$ on which the two topologies $\mathfrak{p}$ and $\tau$ coincide (this can be done by (8.38) in Kechris [11]). Then by going to a smaller E-invariant $\mathfrak{p}$-dense $G_{\delta}$ set $B \subset A \cap Y$, we observe that $B$ is $g_{n}$-invariant for each $n$, so $g_{n}$ is a homeomorphism of $B$.

LEMMA 12. Let $G$ be a countable group of homeomorphisms of a perfect Polish space $X$ such that the induced equivalence relation $\mathrm{E}=\mathrm{E}_{G}$ is generically ergodic. Then there is a dense G-orbit.

Proof. Notice that the orbit of $x$ is dense iff $G . x$ intersects every nonempty open set iff $x \in G$.U for every non-empty open set $U$. But as $\mathrm{E}$ is generically ergodic, $[U]_{\mathrm{E}}=G . U$ is open dense for every non-empty open $U$. Therefore, if $x \in \bigcap_{n} G . U_{n}$, where $\left\{U_{n}\right\}_{\mathbb{N}}$ is a basis of non-empty open sets, the orbit of $x$ is dense.

We can now state our theorem.

THEOREM 13. Let E be a non-singular, generically ergodic countable Borel equivalence relation on a perfect Polish space $X$ and let $H$ be a group such that $[\mathrm{E}]^{*} \leq H \leq \operatorname{Aut}(\mathrm{CAT}(X))$. Then $H$ carries no Polish group topology.

Corollary 14 (G. Hjorth [6, p. 153]). There is no Polish group topology on the automorphism group of the category algebra of $\mathbb{R}$.

We will need the following elementary lemma. 
LEMma 15. Let $\mathrm{E}$ be a non-singular, generically ergodic countable Borel equivalence relation on a perfect Polish space $X$ and suppose $A, B$ are elements of $\mathrm{CAT}(X)$ different from $\emptyset$ and $X$. Then there is an element $g \in[\mathrm{E}]^{*}$ such that $g(A)=B$ and $\neg(A \vee B) \in \operatorname{stab}(g)$.

Let us prove the theorem given the lemma.

Proof of Theorem 13. By the remarks above, we know that $\operatorname{CAT}(X)$ is a homogeneous complete boolean algebra containing a countable dense subalgebra. It is also clear by the lemma that $G=[\mathrm{E}]^{*}$ satisfies conditions (i)-(iii) of Proposition 2, whence the same holds for $H$. So any Hausdorff, Baire topology on $H$ must extend the topology of pointwise convergence on $\operatorname{CAT}(X)$. That is, for any $A, B \in \operatorname{CAT}(X)$, the set $\{h \in H \mid h(A)=B\}$ is open. But as $H$ acts transitively on $\operatorname{CAT}(X)$ and the latter is uncountable, we get a continuum of disjoint translates of some non-empty open set, whence the uniform Suslin number of $H$ is $\left(2^{\aleph_{0}}\right)^{+}$. So no topology on $H$ can be Polish.

Proof of Lemma 15. We can suppose that $A$ and $B$ are non-meagre, non-comeagre Borel subsets of $X$ and we want to construct some $g \in[\mathrm{E}]$ such that $g(A)$ differs from $B$ only in a meagre set.

Let $G$ be a countable group of Borel automorphisms of $X$ inducing E. Find an invariant dense $G_{\delta}$ subset $X_{0} \subset X$ such that

- $X_{0}$ is zero-dimensional.

- $G$ acts by homeomorphisms on $X_{0}$.

- $A_{0}=A \cap X_{0}$ and $B_{0}=B \cap X_{0}$ are clopen in $X_{0}$.

- Every $G$-orbit is dense.

Moreover, let $Y_{0}=A_{0} \cup B_{0}$ and $\left\{x_{n}\right\}$ be some dense subset of $Y_{0}$. We let $\mathbb{P}$ be the set of all homeomorphisms $f$ such that $\operatorname{dom}(f)$ and $\operatorname{rg}(f)$ are clopen subsets of $Y_{0}$,

$$
\forall x \in \operatorname{dom}(f)\left[x \mathrm{E} f(x) \&\left(x \in A_{0} \leftrightarrow f(x) \in B_{0}\right)\right]
$$

and $A_{0} \nsubseteq \operatorname{dom}(f), Y_{0} \backslash A_{0} \nsubseteq \operatorname{dom}(f), B_{0} \nsubseteq \operatorname{rg}(f), Y_{0} \backslash B_{0} \nsubseteq \operatorname{rg}(f)$. Order $\mathbb{P}$ by reverse inclusion, i.e., $g \leq f \Leftrightarrow g$ extends $f$ as a function. Now put

$$
\mathbb{D}_{n}=\left\{f \in \mathbb{P} \mid x_{n} \in \operatorname{dom}(f)\right\}, \quad \mathbb{T}_{n}=\left\{f \in \mathbb{P} \mid x_{n} \in \operatorname{rg}(f)\right\} .
$$

We claim that $\mathbb{D}_{n}$ and $\mathbb{T}_{n}$ are dense in $\mathbb{P}$ for each $n \in \mathbb{N}$. Let us just show this for $\mathbb{D}_{n}$. So suppose $f \in \mathbb{P}$ is given such that $x_{n} \notin \operatorname{dom}(f)$. Then we can find some $\gamma \in G$ such that $\gamma\left(x_{n}\right) \notin \mathrm{rg}(f)$ and $x_{n} \in A_{0} \leftrightarrow \gamma\left(x_{n}\right) \in B_{0}$. For if $x_{n} \in A_{0}$, notice that $B_{0} \backslash \operatorname{rg}(f)$ is a non-empty clopen set, so as the orbit of $x_{n}$ is dense, there is a $\gamma$ such that $\gamma\left(x_{n}\right) \in B_{0} \backslash \operatorname{rg}(f)$. Similarly if $x_{n} \notin A_{0}$. So using the continuity of $\gamma$, we can find clopen $U, V \subseteq Y_{0}$ such that $x_{n} \in U, U \cap \operatorname{dom}(f)=\emptyset, V \cap \operatorname{rg}(f)=\emptyset, \gamma(U)=V$, and $A_{0} \nsubseteq U \cup \operatorname{dom}(f)$, $Y_{0} \backslash A_{0} \nsubseteq U \cup \operatorname{dom}(f), B_{0} \nsubseteq V \cup \operatorname{rg}(f), Y_{0} \backslash B_{0} \nsubseteq V \cup \operatorname{rg}(f)$. Then $f \cup \gamma \uparrow_{U} \in \mathbb{D}_{n}$. 


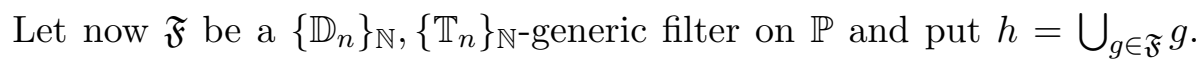
Then $h$ is easily seen to be a homeomorphism of dense open subsets $U=$ $\operatorname{dom}(h)$ and $V=\operatorname{rg}(h)$ of $Y_{0}$. Moreover,

$$
\forall x \in U\left[h(x) \mathrm{E} x \&\left(x \in A_{0} \leftrightarrow h(x) \in B_{0}\right)\right] .
$$

Then $g=h \cup \mathrm{id} \uparrow_{X_{0} \backslash Y_{0}}$ is defined on a dense $G_{\delta}$ subset of $X$ such that

$$
\forall^{*} x \in X[g(x) \mathrm{E} x \&(x \in A \leftrightarrow g(x) \in B)] .
$$

Now, using the fact that any comeagre subset of $X$ contains an E-invariant dense $G_{\delta}$ subset we see that $g$ can be modified on an invariant meagre set so as to be in $[E]$, which finishes the proof.

A theorem due to Sullivan, Weiss and Wright [20] says that if $E$ and $F$ are non-singular, generically ergodic countable Borel equivalence relations on perfect Polish spaces $X$ and $Y$ respectively, then there are invariant dense $G_{\delta}$ subsets $X_{0} \subseteq X$ and $Y_{0} \subseteq Y$ and a homeomorphism $\phi: X_{0} \leftrightarrow Y_{0}$ such that $\forall x, x^{\prime} \in X_{0}\left(x \mathrm{E} x^{\prime} \leftrightarrow \phi(x) \mathrm{F} \phi\left(x^{\prime}\right)\right)$. So in particular $\phi$ induces an isomorphism of $[\mathrm{E}]^{*}$ with $[\mathrm{F}]^{*}$. But another natural question is whether any isomorphism between $[E]^{*}$ and $[F]^{*}$ lifts to such a point map. The answer to this question turns out to be contained in a very general result of M. Rubin on the reconstruction of boolean algebras from their automorphism groups.

Definition 16 . Let $\mathcal{K}$ be a class of pairs $(\mathfrak{B}, G)$, where $\mathfrak{B}$ is a boolean algebra and $G \leq \operatorname{Aut}(\mathfrak{B})$. Then $\mathcal{K}$ is said to be faithful if for all $\left(\mathfrak{B}_{0}, G_{0}\right)$, $\left(\mathfrak{B}_{1}, G_{1}\right) \in \mathcal{K}$ and isomorphisms $\Theta: G_{0} \cong G_{1}$ there is an isomorphism $\phi: \mathfrak{B}_{0} \cong \mathfrak{B}_{1}$ such that $\Theta(g)=\phi \circ g \circ \phi^{-1}$ for all $g \in G_{0}$.

Definition 17. Let $\mathfrak{B}$ be a complete atomless boolean algebra and $G \leq \operatorname{Aut}(\mathfrak{B})$. The pair $(\mathfrak{B}, G)$ is said to be a local movement system if the set $\{\operatorname{SUPP}(g) \mid g \in G\}$ is dense in $\mathfrak{B}$, where we define $\operatorname{SUPP}(g)=\bigvee\{a \in \mathfrak{B} \mid$ $g(a) \wedge a=0\}$ (notice that this makes sense as we are working in a complete boolean algebra).

Theorem 18 (M. Rubin [15]). The class of local movement systems is faithful.

Lemma 19. Let $\left(X, \mathfrak{p}_{X}\right)$ and $\left(Y, \mathfrak{p}_{Y}\right)$ be Polish spaces and $\phi: X \leftrightarrow Y$ a Borel isomorphism preserving meagre sets. Then there are dense $G_{\delta}$ subsets $X_{0} \subseteq X$ and $Y_{0} \subseteq Y$ such that $\phi$ is a homeomorphism of $X_{0}$ with $Y_{0}$.

Proof. Find finer Polish topologies $\tau_{X}$ and $\tau_{Y}$ such that $\phi$ is a homeomorphism of $\left(X, \tau_{X}\right)$ with $\left(Y, \tau_{Y}\right)$. Then there are $\mathfrak{p}_{X}$-dense $G_{\delta}$ and $\mathfrak{p}_{Y}$-dense $G_{\delta}$ sets $A \subseteq X$ and $B \subseteq Y$ such that $\mathfrak{p}_{X} \uparrow_{A}=\tau_{X} \uparrow_{A}$ and $\mathfrak{p}_{Y} \uparrow_{B}=\tau_{Y} \uparrow_{B}$. But as $\mathfrak{p}_{X} \subseteq \tau_{X}$ and $\mathfrak{p}_{Y} \subseteq \tau_{Y}, A$ and $B$ are also $G_{\delta}$ in $\tau_{X}$ and $\tau_{Y}$. Moreover, $\phi(A)$ and $\phi^{-1}(B)$ are $\tau_{Y^{-}} G_{\delta}$ and $\tau_{X^{-}} G_{\delta}$ respectively, as $\phi$ is a homeomorphism with respect to these topologies. Therefore, $X_{0}=A \cap \phi^{-1}(B)$ is $G_{\delta}$ 
in $\left(A, \mathfrak{p}_{X}\right)=\left(A, \tau_{X}\right)$ and $Y_{0}=B \cap \phi(A)$ is $G_{\delta}$ in $\left(B, \mathfrak{p}_{Y}\right)=\left(B, \tau_{Y}\right)$. But as $\phi$ also preserves meagre sets, they are comeagre in $\left(X, \mathfrak{p}_{X}\right)$ and $\left(Y, \mathfrak{p}_{Y}\right)$ respectively and hence dense $G_{\delta}$ in $\left(X, \mathfrak{p}_{X}\right)$ and $\left(Y, \mathfrak{p}_{Y}\right)$.

COROllary 20. Suppose $\mathrm{E}$ and $\mathrm{F}$ are non-singular, generically ergodic countable Borel equivalence relations on perfect Polish spaces $X$ and $Y$ respectively. Then

(i) $\operatorname{Aut}\left([\mathrm{E}]^{*}\right)$ is equal to the normaliser of $[\mathrm{E}]^{*}$ in $\operatorname{Aut}(\mathrm{CAT}(X))$.

(ii) If $\Theta:[\mathrm{E}]^{*} \cong[\mathrm{F}]^{*}$ is an isomorphism, then there are invariant dense $G_{\delta}$ subsets $X_{0} \subseteq X$ and $Y_{0} \subseteq Y$ and a homeomorphism $\phi: X_{0} \leftrightarrow Y_{0}$ such that $\Theta(g)=\phi \circ g \circ \phi^{-1}$.

Proof. Again we consider $[\mathrm{E}]^{*}$ to be a subgroup of $\operatorname{Aut}(\mathrm{CAT}(X))$, and we claim that $\left(\operatorname{CAT}(X),[\mathrm{E}]^{*}\right)$ is a local movement system. So suppose $C \subseteq X$ is a non-meagre Borel set; we need to find $g \in[\mathrm{E}]^{*}$ such that $\operatorname{SUPP}(g) \leq C$. But for this let $A \vee B=C$ be a partition of $C$ into non-meagre Borel sets and find by Lemma 15 some $g \in[\mathrm{E}]^{*}$ such that $g(A)=B$ and $\neg C \in \operatorname{stab}(g)$. That is, $\operatorname{SUPP}(g)=C$. This shows $\left(\operatorname{CAT}(X),[\mathrm{E}]^{*}\right)$ to be a local movement system, whence any automorphism of $[\mathrm{E}]^{*}$ is induced by an automorphism of $\operatorname{CAT}(X)$. So $\operatorname{Aut}\left([\mathrm{E}]^{*}\right)$ is equal to the normaliser, $N\left([\mathrm{E}]^{*}\right)$, of $[\mathrm{E}]^{*}$ in $\operatorname{Aut}(\operatorname{CAT}(X))$.

For (ii) we can apply the same reasoning and then notice that any isomorphism between the category algebras of two Polish spaces is induced by a homeomorphism between dense $G_{\delta}$ subsets (this follows from Lemma 19 and from (15.10) in [11]).

We should in this connection give an explicit description of the normaliser of $[\mathrm{E}]^{*}$ in $\operatorname{Aut}(\operatorname{CAT}(X))$ :

$$
\begin{aligned}
& N\left([\mathrm{E}]^{*}\right)=\{g \in \operatorname{Aut}(\mathrm{CAT}(X)) \mid \\
&\left.\forall^{*} x \forall y \in[x]_{\mathrm{E}}\left(g(x) \mathrm{E} g(y) \& g^{-1}(x) \mathrm{E} g^{-1}(y)\right)\right\} .
\end{aligned}
$$

To see this, notice first that if

$$
\forall^{*} x \forall y \in[x]_{\mathrm{E}}\left(g(x) \mathrm{E} g(y) \& g^{-1}(x) \mathrm{E} g^{-1}(y)\right)
$$

and $f \in[\mathrm{E}]^{*}$, then

$$
\forall^{*} y\left(g^{-1} f(y) \mathrm{E} g^{-1}(y)\right)
$$

and hence

$$
\forall^{*} x\left(g^{-1} f g(x) \mathrm{E} g^{-1} g(x)=x\right),
$$

whereby $g^{-1} f g \in[\mathrm{E}]^{*}$. Similarly, $g f g^{-1} \in[\mathrm{E}]^{*}$, whence $g \in N\left([\mathrm{E}]^{*}\right)$.

Conversely, fix a countable group $G$ of Borel automorphisms of $X$ inducing E. Now, suppose, e.g., $\exists^{*} x \exists y \in[x]_{\mathrm{E}}(g(x) \neg \mathrm{E} g(y))$. Then for some $\gamma \in G$, there is a non-meagre Borel set $A \subseteq X$ such that $\forall x \in A(g(x) \neg \mathrm{E} g(\gamma \cdot x))$. By going to some smaller non-meagre Borel set, we can suppose that $\gamma \cdot A \cap A=\emptyset$. 
We define $f \in[\mathrm{E}]^{*}$ by

$$
f(x)= \begin{cases}\gamma \cdot x & \text { if } x \in A, \\ \gamma^{-1} \cdot x & \text { if } x \in \gamma \cdot A, \\ x & \text { otherwise. }\end{cases}
$$

Then for $y=g(x) \in g(A)$,

$$
g f g^{-1}(y)=g f g^{-1}(g(x))=g f(x)=g(\gamma \cdot x) \neg \mathrm{E} g(x)=y .
$$

So for a non-meagre set of $y \in X, g f g^{-1}(y) \neg \mathrm{E} y$, whence $g f g^{-1} \notin[\mathrm{E}]^{*}$ and $g \notin N\left([\mathrm{E}]^{*}\right)$.

One might wonder what happens if in Theorem 13 we consider the automorphism group of the measure algebra of a Lebesgue probability space, i.e., the group of non-singular Borel automorphisms, where two automorphisms are identified if they agree on a conull set. It turns out that in this case there is indeed a Polish group topology (see [11, 17.46]). So what goes wrong here? The answer is of course that the measure algebra, in contradistinction to the category algebra, does not have a countable dense subalgebra, so Proposition 2 does not apply.

We should also mention that $\mathrm{H}$. Woodin has proved that there is no Polish topology on the category algebra of $\mathbb{R}$ that renders the boolean operations continuous (see [17] for a simple proof of this fact). For the measure algebra of a Lebesgue probability space the situation is again different (see $[11,17.43])$.

4. Homomorphisms into locally compact groups. The above-mentioned paper by Gaughan [5] was in answer to a question of Ulam on whether $\mathcal{S}_{\infty}$ could be made into a locally compact Polish group (and therefore carry a Haar measure). Since $\mathcal{S}_{\infty}$ has a unique Polish group topology this is not the case. But, as we shall see, it does not even embed into such a group, and in fact any (abstract) homomorphism of $\mathcal{S}_{\infty}$ into a locally compact Polish group is trivial.

Proposition 21. Let $\pi: \mathcal{S}_{\infty} \rightarrow H$ be a non-trivial abstract homomorphism into a separable Hausdorff group. Then $\pi$ is a homeomorphism onto a closed subgroup of $H$.

Proof. A result of [12] implies that any homomorphism from $\mathcal{S}_{\infty}$ into a separable group is automatically continuous, so as $H$ is Hausdorff, ker $\pi$ is a closed normal subgroup of $\mathcal{S}_{\infty}$. Thus as $\mathcal{S}_{\infty}$ is topologically simple this means that $\pi$ is either trivial, contradicting our assumption, or an embedding. Therefore, as $H$ is Hausdorff, so is the induced topology on $\pi$ " $\mathcal{S}_{\infty}$. But by results of Gaughan [5], the Polish topology on $\mathcal{S}_{\infty}$ is the minimal Hausdorff group topology, whence $\pi$ is also an open map, and hence $\pi$ is a homeomorphism onto the subgroup $\pi$ " $\mathcal{S}_{\infty}$ of $H$. We now only need to 
check that $\pi " S_{\infty}$ is also closed in $H$. So suppose that $\left(g_{\lambda}\right)$ is a net in $\pi " S_{\infty}$ converging to a point $h \in H$. Then $\left(g_{\lambda}\right)$ is both left and right Cauchy, so by the completeness of $\pi " S_{\infty}$ must also converge in $\pi " S_{\infty}$, i.e. $h \in \pi " S_{\infty}$. So $\pi " S_{\infty}$ is closed.

We shall see that a weaker result actually holds for a much larger class of permutation groups. Before we begin we need to consider some new concepts.

Let $G$ be any group and consider its action on $G^{n}(n \geq 1)$ given by

$$
g \cdot\left(h_{1}, \ldots, h_{n}\right)=\left(g h_{1} g^{-1}, \ldots, g h_{n} g^{-1}\right) .
$$

So when $n=1$ this is just the usual action by conjugation. A Polish group is said to have ample generics in case there is a comeagre orbit for the above action of $G$ on $G^{n}$ for each $n \geq 1$. This is in general stronger than just requiring that $G$ should have a comeagre conjugacy class. In fact, one can show that in this case any homomorphism into a topological group with uniform Suslin number $\leq 2^{\aleph_{0}}$ is automatically continuous [12].

It is now known that a fairly large class of automorphism groups have ample generics. These include the automorphism groups of (i) $\omega$-stable, $\omega$ categorical structures [7], (ii) the random graph [9], (iii) the rational Urysohn metric space [18], and (iv) the group of measure preserving homeomorphisms of $2^{\mathbb{N}}$ [12]. So in particular it holds for $\mathcal{S}_{\infty}$.

Incidentally, all of these groups also have a neighbourhood basis at the identity consisting of clopen subgroups with a dense (in fact comeagre) conjugacy class [12].

A topological group is called a pro-Lie group if it is a projective limit of Lie groups (which is equivalent to being isomorphic to a closed subgroup of a product of Lie groups). In a personal communication, Karl H. Hofmann has shown that no non-trivial Lie group can have a dense conjugacy class, and this in turn implies that no non-trivial pro-Lie group can have a dense conjugacy class.

TheOREm 22. Let $G$ be a Polish group with ample generics and suppose that $G$ has a neighbourhood basis at the identity consisting of open subgroups with dense conjugacy classes. Then there is no non-trivial abstract homomorphism of $G$ into a locally compact Hausdorff group with uniform Suslin number $\leq 2^{\aleph_{0}}$ (e.g., into a separable group).

Proof. Suppose $H$ is a locally compact Hausdorff group with uniform Suslin number $\leq 2^{\aleph_{0}}$ and let $\pi: G \rightarrow H$ be any homomorphism. As $G$ has ample generics, $\pi$ is necessarily continuous. Put $N=\overline{\pi " G} \leq H$, which is still locally compact. Let $N_{0} \leq N$ be the connected component of the identity element in $N$, whereby $N_{0}$ is a closed normal subgroup and $N / N_{0}$ a totally disconnected locally compact Hausdorff group. Therefore, by a theorem of van Dantzig (Theorem 1.34 in [8]), $N / N_{0}$ has a neighbourhood basis at the 
identity consisting of compact open subgroups. So choose some compact open $K \leq N / N_{0}$ and denote by $\pi_{0}$ the composition of $\pi$ with the quotient mapping from $N$ to $N / N_{0}$. Then $\pi_{0}^{-1}(K)$ is open in $G$ and we can therefore find a clopen $F \leq \pi_{0}^{-1}(K) \leq G$ with a dense conjugacy class $C \subset F$. But then $\pi_{0}(C)$ is a dense set in $\overline{\pi_{0} " F}$ and is contained in a single conjugacy class of $\overline{\pi_{0} " F}$. Hence as this latter group is compact and the conjugation action on itself is continuous, each conjugacy class is closed. Therefore the conjugacy class of the identity element is everything, i.e., $\overline{\pi_{0} " F}=\{e\}$. So $F \leq \operatorname{ker} \pi_{0}$ is open and as $\operatorname{ker} \pi_{0}$ is normal in $G$, it is conjugacy invariant. Since $G$ has ample generics, it in particular has a dense conjugacy class, so $\operatorname{ker} \pi_{0}=G$.

This shows that $\pi$ " $G \leq N_{0}$ and as $G$ is dense in $N$ and $N_{0}$ is closed, $N_{0}=N$. So $N$ is connected and therefore by the theorem of Gleason, Montgomery, Yamabe and Zippin (see [2]) it is pro-Lie. Now, the image of any dense conjugacy class in $G$ under $\pi$ will be a dense set in $N$ contained in a single conjugacy class, which, by the theorem of Hofmann mentioned above, implies that $N=\{e\}$, whence $\pi " G=\{e\}$.

So from either Proposition 21 or Theorem 22 follows:

Corollary 23. Any abstract homomorphism of $\mathcal{S}_{\infty}$ into a locally compact Polish group is trivial.

The above results imply that a great number of groups appearing in analysis cannot be abstractly embedded into a locally compact Polish group. For this it is enough that they should contain some copy of $\mathcal{S}_{\infty}$ or indeed any of the other groups mentioned to have ample generics. So these remarks apply in particular to the unitary group of $\ell_{2}$ or the group of isomorphisms of $\ell_{p}$.

\section{References}

[1] S. Bezuglyŭ, A. H. Dooley and J. Kwiatkowski, Topologies on the group of Borel automorphisms of a standard Borel space, arXiv:math.DS/0410506.

[2] H. G. Dales, P. Aiena, J. Eschmeier, K. Laursen and G. A. Willis, Introduction to Banach Algebras, Operators, and Harmonic Analysis, London Math. Soc. Stud. Texts 57, Cambridge Univ. Press, Cambridge, 2003.

[3] R. M. Dudley, Continuity of homomorphisms, Duke Math. J. 28 (1961), 587-594.

[4] H. A. Dye, On groups of measure preserving transformations. II, Amer. J. Math. 85 (1963), 551-576.

[5] E. D. Gaughan, Topological group structures of infinite symmetric groups, Proc. Nat. Acad. Sci. U.S.A. 58 (1967), 907-910.

[6] G. Hjorth, Classification and Orbit Equivalence Relations, Math. Surveys Monogr. 75, Amer. Math. Soc., Providence, RI, 2000. 
[7] W. Hodges, I. Hodkinson, D. Lascar, and S. Shelah, The small index property for $\omega$-stable $\omega$-categorical structures and for the random graph, J. London Math. Soc. (2) 48 (1993), 204-218.

[8] K. H. Hofmann and S. A. Morris, The Structure of Compact Groups. A Primer for the Student-a Handbook for the Expert, de Gruyter Stud. Math. 25, de Gruyter, Berlin, 1998.

[9] E. Hrushovski, Extending partial isomorphisms of graphs, Combinatorica 12 (1992), 4, 411-416.

[10] R. R. Kallman, Uniqueness results for homeomorphism groups, Trans. Amer. Math. Soc. 295 (1986), 389-396.

[11] A. S. Kechris, Classical Descriptive Set Theory, Grad. Texts in Math. 156, Springer, New York, 1995.

[12] A. S. Kechris and C. Rosendal, Turbulence, amalgamation and generic automorphisms of homogeneous structures, arXiv:math.LO/0409567.

[13] R. D. Mauldin (ed.), The Scottish Book. Mathematics from the Scottish Café. Including selected papers presented at the Scottish Book Conference held at North Texas State University, Denton, Tex., May 1979, Birkhäuser, Boston, 1981.

[14] M. G. Nadkarni, Basic Ergodic Theory, 2nd ed., Birkhäuser Advanced Texts: Basler Lehrbücher, Birkhäuser, Basel, 1998.

[15] M. Rubin, Locally moving groups and reconstruction problems, in: Ordered Groups and Infinite Permutation Groups, Math. Appl. 354, Kluwer, Dordrecht, 1996, 121157.

[16] S. Shelah, Can you take Solovay's inaccessible away? Israel J. Math. 48 (1984), $1-47$.

[17] S. Solecki, Polish group topologies, in: Sets and Proofs (Leeds, 1997), London Math. Soc. Lecture Note Ser. 258, Cambridge Univ. Press, Cambridge, 1999, 339-364.

[18] —, Extending partial isometries, preprint.

[19] R. M. Solovay, A model of set-theory in which every set of reals is Lebesgue measurable, Ann. of Math. (2) 92 (1970), 1-56.

[20] D. Sullivan, B. Weiss and J. D. M. Wright, Generic dynamics and monotone complete $C^{*}$-algebras, Trans. Amer. Math. Soc. 295 (1986), 795-809.

Mathematics 253-37

California Institute of Technology

Pasadena, CA 91125, U.S.A.
Current address:

Department of Mathematics University of Illinois at Urbana-Champaign 1409 W. Green Street (MC-382) Urbana, IL 61801-2975, U.S.A. E-mail: rosendal@math.uiuc.edu

Received 18 January 2005;

in revised form 2 September 2005 